DAMTP-1999-181

astro-ph/0004098

\title{
Cosmic Magnetic Fields From Particle Physics"
}

\author{
Ola Törnkvist用 \\ Department of Applied Mathematics and Theoretical Physics, \\ Centre for Mathematical Sciences, University of Cambridge, \\ Wilberforce Road, Cambridge CB3 0WA, United Kingdom
}

31 March 2000

\begin{abstract}
I review a number of particle-physics models that lead to the creation of magnetic fields in the early universe and address the complex problem of evolving such primordial magnetic fields into the fields observed today. Implications for future observations of the Cosmic Microwave Background (CMB) are briefly discussed.
\end{abstract}

*Talk presented at the 7th International Symposium on Particles, Strings and Cosmology (PASCOS99) at Granlibakken, Lake Tahoe, 10 - 16 Dec 1999, to appear in the proceedings.

${ }^{\ddagger}$ E-mail: o.tornkvist@damtp.cam.ac.uk 


\section{Introduction to Cosmic Magnetic Fields}

Magnetic fields have been observed in a large number of spiral galaxies, including the Milky Way [1]. In almost all cases, the galactic field strengths are measured to be a few times $10^{-6} \mathrm{G}$. This particular value has also been found at a redshift of $z=0.395$ [2] and between the galaxies in clusters. In spiral galaxies, one discovers that the magnetic field is aligned with the spiral arms and density waves in the disk. A plausible explanation is that it was created by a mean-field dynamo mechanism [3], in which a much smaller seed field was exponentially amplified by the turbulent motion of ionised gas in conjunction with the differential rotation of the galaxy.

For the dynamo to work, the initial seed field must be correlated on a scale of 100 pc, corresponding to the largest turbulent eddy [3]. The required strength of the seed field is subject to large uncertainties; past authors have quoted $10^{-21 \pm 2} \mathrm{G}$ as the lower bound at the time of completed galaxy formation. This would present a problem for most particle-physics and field-theory inspired mechanisms of magnetic field generation. However, in recent work with A.-C. Davis and M.J. Lilley [ [4], I have shown that the lower bound on the dynamo seed field can be significantly relaxed if the universe is flat with a cosmological constant, as is suggested by recent supernovae observations [5]. In particular, for the same dynamo parameters that give a lower bound of $10^{-20} \mathrm{G}$ for $\Omega_{0}=1, \Omega_{\Lambda}=0$, we obtain $10^{-30} \mathrm{G}$ for $\Omega_{0}=0.2=1-\Omega_{\Lambda}$, implying that particle-physics mechanisms could still be viable. The observation at redshift $z=0.395$ [2] can also be accounted for with these parameters, but requires a seed field of at least $10^{-23} \mathrm{G}$ [4].

The magnetic field is amplified by the dynamo until its energy reaches equipartition with the kinetic energy of the ionised gas, $\left\langle B^{2} / 2\right\rangle=\left\langle\rho v^{2} / 2\right\rangle$. Thus a final field of $B_{0} \approx 10^{-6} \mathrm{G}$ results for any seed field of sufficient strength.

It is difficult to explain the galactic field strength without a dynamo mechanism. To this end, one would require a strong primordial field of $10^{-3}\left(\Omega_{0} h^{2}\right)^{1 / 3} \mathrm{G}$ at the epoch of radiation decoupling $t_{\mathrm{dec}}$, corresponding to a field strength $10^{-9}\left(\Omega_{0} h^{2}\right)^{1 / 3} \mathrm{G}$ on comoving scales of $1 \mathrm{Mpc}$. Future precision measurements of the CMB will put severe constraints on such a primordial field [6]. Moreover, magnetic fields on Mpc scales have been probed by observations of the Faraday rotation of polarised light from distant luminous sources, which give an upper bound of about $10^{-9} \mathrm{G}$ [1]. The observation of micro-Gauss fields between galaxies in clusters presents an interesting dilemma. Because such regions are considerably less dense than galaxies, it is doubtful whether a dynamo could have been operative. Thus the intra-cluster magnetic fields, unless somehow ejected from galaxies, have formed directly from a primordial field stronger than $10^{-3}\left(\Omega_{0} h^{2}\right)^{1 / 3} \mathrm{G}$ at $t_{\mathrm{dec}}$. Such a field would certainly leave a signature in future CMB data [6].

Although particle-physics inspired models typically produce weak seed fields, they 
tend to give precise predictions, unlike many astrophysical mechanisms, where the magnetic field strength is determined by complicated nonlinear dynamics, or solutions of general relativity with a magnetic field [7], where the field strength must be fixed by observations. With the possible exception of the last-mentioned model, there is no compelling scenario that produces a primordial field strong enough to eliminate the need for a dynamo.

Seed fields for the dynamo can be astrophysical or primordial. In the former category there is the important possibility that a seed field may arise spontaneously due to nonparallel gradients of pressure and charge density during the collapse of a protogalaxy [8]. For the rest of this talk, however, I shall assume that the seed field is primordial.

\section{Primordial Seed Fields}

It is useful to distinguish between primordial seed fields that are produced with correlation length smaller than vs. larger than the horizon size.

Subhorizon-scale seed fields typically arise in first-order phase transitions and from causal processes involving defects. For example, magnetic fields may be created on the surface of bubble walls [9] due to local charge separation induced by baryon-number gradients. The magnetic fields are then amplified by plasma turbulence near the bubble wall. This possibility has been explored for the QCD [10] as well as for the electroweak [11] phase transition.

The production of magnetic fields in collisions of expanding true-vacuum bubbles will be discussed in Sec. $⿴$. Fields can also be generated in the wakes of, or due to the wiggles of, GUT-scale cosmic strings during structure formation, resulting in a large correlation length [12]. Joyce and Shaposhnikov have shown that an asymmetry of right-handed electrons, possibly generated at the GUT scale, would become unstable to the generation of a hypercharge magnetic field shortly before the electroweak phase transition [13], leading to a correlation length of order $10^{6} / T$.

Horizon-scale seed fields emerge naturally in second-order phase transitions of gauge theories from the failure of covariant derivatives of the Higgs field to correlate on superhorizon scales [14].

Superhorizon-scale seed fields can arise as a solution of the Einstein equations for axisymmetric universes [7] and in inflationary or pre-Big Bang (superstring) scenarios. In the latter case, vacuum fluctuations of the field tensor are amplified by the dynamical dilaton field [15]. Inflationary models produce extremely weak magnetic fields unless conformal invariance is explicitly broken [16], but even then great difficulties remain. An exciting new possibility is that magnetic fields may be produced via parametric resonance with an oscillating field [17] e.g. during preheating after inflation. Because the inflaton 
is initially coherent on superhorizon scales, large correlations can arise without violating causality. A similar proposal involves charged scalar particles, minimally coupled to gravity, that are created from the vacuum due to the changing space-time geometry at the end of inflation. The particles give rise to fluctuating electric currents which are claimed to produce superhorizon-scale (indeed, galactic-scale) fields of sufficient strength to satisfy the galactic dynamo bound [18]. This mechanism deserves further investigation.

\section{The Evolution of Primordial Magnetic Fields}

Many particle-physics and field-theory scenarios for producing primordial magnetic fields result in too small a correlation length $\xi$, which is a serious problem. If the fields are produced at the QCD phase transition or earlier with sub-horizon correlation length, then the expansion of the universe cannot stretch $\xi$ to more than 1 pc today (see Fig. 1). This is far short of the galactic dynamo lower bound of 5-10 kpc (comoving), corresponding to $100 \mathrm{pc}$ in a virialised galaxy [4].

Nevertheless, it has been argued by many authors [19, 20, 21, 22 that the correlation length will grow more rapidly due to magnetohydrodynamic (MHD) turbulence and inverse cascade, which transfers power from small-scale to large-scale Fourier modes. In Fig. 1, several non-relativistic models for this evolution are analysed. The most conservative estimate is obtained by assuming that the magnetic field strength on the scale of one correlation length at any time equals the volume average of fields that were produced on smaller scales but have since decayed [20]. This leads to a growth $\xi \sim t^{7 / 10}$ (obtained from the Minkowski-space growth $\xi \sim t^{2 / 5}$ via the substitution $t \rightarrow \tau=t^{1 / 2}$ and multiplication by the scale factor). The most optimistic estimate corresponds to the case when the magnetic field has maximal helicity in relation to the energy density [20, 21]. As magnetic helicity is approximately conserved in the high-conductivity early-universe environment, one obtains the growth law $\xi \sim t^{5 / 6}$. Turbulence ends, freezing the growth (in comoving coordinates) when the kinetic Reynolds number drops below unity at the $e^{+} e^{-}$annihilation or later, depending on the model and the parameters of the initial field.

An intermediate and rather plausible estimate has been given by Dimopoulos and Davis [22], who use the fact that the magnetic flux enclosed by a (sufficiently large) comoving closed curve is conserved. The correlation length here increases at a rate given by the Alfvén velocity, so that $\xi \sim t^{3 / 4}$.

As Fig. 1 shows, only the most optimistic of these growth laws leads to a correlation length today that satisfies the galactic dynamo bound. This occurs for fields correlated over the horizon scale at the QCD phase transition. Beware, however, that the growth laws were derived using non-relativistic MHD equations assuming that the magnetic field energy density remains in equipartition with the kinetic energy density $\rho \bar{v}^{2} / 2$, where $\bar{v}$ 


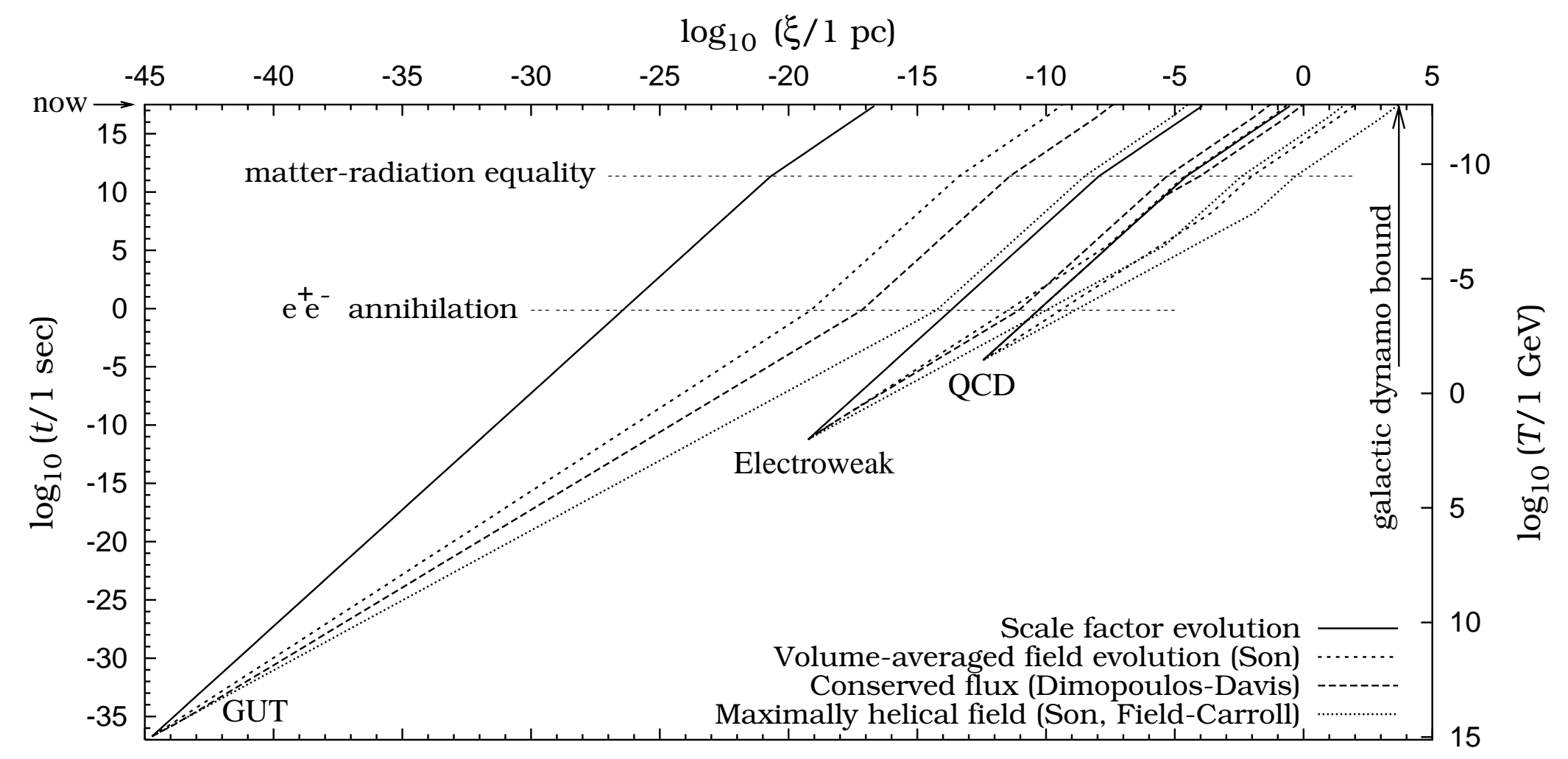

Figure 1: Four models of evolution of the correlation length $\xi(t)$ of primordial fields generated at three different epochs $t_{\mathrm{i}}$ with $\xi\left(t_{\mathrm{i}}\right)=t_{\mathrm{i}}$ (horizon size). 
is the presumed non-relativistic "bulk velocity" of the ultra-relativistic plasma. It seems plausible that a relativistic treatment could alter the predicted evolution dramatically. In this light, I find it too early to reject the idea that also subhorizon fields might evolve into fields sufficiently correlated to seed the galactic dynamo.

At the same time, Fig. 1 demonstrates the intrinsic advantage of superhorizon field generation mechanisms. For these, the principal problem is not the correlation length, but to achieve sufficient strength of the magnetic field.

\section{Magnetic Fields From Bubble Collisions}

A simple solution for the magnetic field generated in a bubble collision was first obtained in a U(1) model by Kibble and Vilenkin [KV] [23]. We have recently obtained an improved analytical solution for the field evolution in that model [24] using an analytical expression for the bubble-wall profile.

Here I shall restrict attention to non-Abelian bubble collisions, taking the minimal Standard Model as an example. The initial Higgs field in the two bubbles can be written

$$
\Phi_{1}=\exp \left(-i \theta_{0} \boldsymbol{n} \cdot \boldsymbol{\sigma}\right)\left(\begin{array}{c}
0 \\
\rho_{1}(x)
\end{array}\right), \quad \Phi_{2}=\exp \left(i \theta_{0} \boldsymbol{n} \cdot \boldsymbol{\sigma}\right)\left(\begin{array}{c}
0 \\
\rho_{2}(x)
\end{array}\right)
$$

where $\boldsymbol{n}, \theta_{0}$ are constants. As the bubbles collide, non-Abelian currents $j_{k}^{A}=i\left[\Phi^{\dagger} T^{A} D_{k} \Phi-\right.$ $\left.\left(D_{k} \Phi\right)^{\dagger} T^{A} \Phi\right]$ develop across the surface of their intersection, where $T^{A}=\left(g^{\prime} / 2, g \sigma^{a} / 2\right)$, $D_{k}=\partial_{k}-i W_{k}^{A} T^{A}$ and $W_{k}^{A}=\left(Y_{k}, W_{k}^{a}\right)$. This, in turn, leads to a ring-like flux of non-Abelian fields encircling the bubble intersection. The recipe for projecting out the electromagnetic field amongst the non-Abelian fields in an arbitrary gauge has been given elsewhere [25].

In the special cases $\boldsymbol{n}=(0,0, \pm 1)$ and $\boldsymbol{n}=\left(n_{1}, n_{2}, 0\right)$ it is known [26] that the nonAbelian flux consists of pure $\mathrm{Z}$ and pure $\mathrm{W}$ vector fields, respectively. When $n_{3} \neq 0, \pm 1$, both $Z$ and $W$ fields are excited and conspire to generate a non-zero electric current and associated magnetic field. As is expected, the resulting field strength is of the order of $M_{W}^{2} / g$ with a correlation length $\xi \sim M_{W}^{-1}$. However, when plasma friction and conductivity are taken into account, the magnetic field spreads over the interior of a bubble [27] leading to an appreciable increase in correlation length.

\section{Acknowledgments}

The author is supported by the European Commission's TMR programme under Contract No. ERBFMBI-CT97-2697 and by the U.K. PPARC. He is grateful to the Royal Society, inter alii, for travel support. 


\section{References}

[1] P. P. Kronberg, Rep. Prog. Phys. 57, 325 (1994).

[2] P. P. Kronberg and J. J. Perry, Ap. J. 263, 518 (1982); P. P. Kronberg, J. J. Perry and E. L.H. Zukowski, ibid. 387, 528 (1992).

[3] Ya. B. Zeldovich, A. A. Ruzmaikin and D.D. Sokolov, Magnetic Fields in Astrophysics (Gordon and Breach, New York, 1983).

[4] A.-C. Davis, M. Lilley and O. Törnkvist, Phys. Rev. D 60, 021301 (1999).

[5] S. Perlmutter et al., Ap. J. 517, 565P (1999); P. M. Garnavich et al., ibid. 509, 74G (1998).

[6] J. Adams, U. H. Danielsson, D. Grasso, and H. Rubinstein, Phys. Lett. B388, 253 (1996); J. D. Barrow, P. G. Ferreira and J. Silk, Phys. Rev. Lett. 78, 3610 (1997); A. Kosowsky and A. Loeb, Ap. J. 469, 1 (1996).

[7] Ya. B. Zeldovich, Zh. Eksp. Teor. Fiz. 48, 986 (1964) [Sov. Phys. JETP 21, 656 (1965)]; K. Thorne, Bull. Am. Phys. Soc. 11, 340 (1966), Ap. J. 148, 51 (1967).

[8] H. Lesch and M. Chiba, Astron. Astrophys. 297, 305L (1995); R.M. Kulsrud, R. Cen, J.P. Ostriker and D. Ryu, Ap. J. 480, 481 (1997).

[9] C. J. Hogan, Phys. Rev. Lett. 51, 1488 (1983)

[10] B. Cheng and A. V. Olinto, Phys. Rev. D 50, 2421 (1994).

[11] G. Baym, D. Bödeker and L. McLerran, Phys. Rev. D 53, 662 (1996); G. Sigl, A. Olinto and K. Jedamzik, ibid. 55, 4582 (1997).

[12] T. Vachaspati and A. Vilenkin, Phys. Rev. Lett. 67, 1057 (1991); K. Dimopoulos, Phys. Rev. D 57, 4629 (1998).

[13] M. Joyce and M. Shaposhnikov, Phys. Rev. Lett. 79, 1193 (1997).

[14] T. Vachaspati, Phys. Lett. B 265, 258 (1991).

[15] M. Gasperini, M. Giovannini and G. Veneziano, Phys. Rev. Lett. 75, 3796 (1995); D. Lemoine and M. Lemoine, Phys. Rev. D 52, 1955 (1995).

[16] M.S. Turner and L. M. Widrow, Phys. Rev. D 37, 2743 (1988); W. D. Garretson, G. B. Field and S. M. Carroll, ibid. 46, 5346 (1992). 
[17] R. Brustein and D.H. Oaknin, Phys. Rev. D 60, 023508 (1999), Phys. Rev. Lett. 82, 2628 (1999); F. Finelli and A. Gruppuso, hep-ph/0001231.

[18] E.A. Calzetta, A. Kandus, and F.D. Mazzitelli, Phys. Rev. D 57, 7139 (1998); A. Kandus, E.A. Calzetta, F.D. Mazzitelli and C.E.M. Wagner, Phys. Lett. B 472, 287 (2000).

[19] A. Brandenburg, K. Enqvist and P. Olesen, Phys. Rev. D 54, 1291 (1996); T. Shiromizu, Phys. Lett. B 443, 127 (1998).

[20] D. T. Son, Phys. Rev. D 59, 063008 (1999).

[21] G. B. Field and S. M. Carroll, astro-ph/9811206.

[22] K. Dimopoulos and A.-C. Davis, Phys. Lett. B390 87 (1997).

[23] T. W B. Kibble and A. Vilenkin, Phys. Rev. D52, 679 (1995).

[24] E. J. Copeland, P. M. Saffin and O. Törnkvist, hep-ph/9907437, to appear in Phys. Rev. D.

[25] O. Törnkvist, hep-ph/9805255, to be published in Phys. Rev. D.

[26] P. Saffin and E. Copeland, Phys. Rev. D 56, 1215 (1997); D. Grasso and A. Riotto, Phys. Lett. B 418, 258 (1998).

[27] A.-C. Davis and M. Lilley, Phys. Rev. D 61, 043502 (2000). 